Clinical Study

\title{
Beneficial Effects of Combining Computed Tomography Enteroclysis/Enterography with Capsule Endoscopy for Screening Tumor Lesions in the Small Intestine
}

\author{
Hiroaki Shibata, ${ }^{1}$ Shinichi Hashimoto, ${ }^{1}$ Kensaku Shimizu, ${ }^{2}$ Ryo Kawasato, ${ }^{1}$ \\ Tomohiro Shirasawa, ${ }^{1}$ Takayuki Yokota, ${ }^{1}$ Hideko Onoda, ${ }^{2}$ Takeshi Okamoto, ${ }^{1}$ \\ Jun Nishikawa, ${ }^{1}$ Naofumi Matsunaga, ${ }^{2}$ and Isao Sakaida ${ }^{1}$ \\ ${ }^{1}$ Department of Gastroenterology and Hepatology, Yamaguchi University Graduate School of Medicine, \\ 1-1-1 Minami-Kogushi, Ube, Yamaguchi 755-8505, Japan \\ ${ }^{2}$ Department of Radiology, Yamaguchi University Graduate School of Medicine, 1-1-1 Minami-Kogushi, \\ Ube, Yamaguchi 755-8505, Japan \\ Correspondence should be addressed to Shinichi Hashimoto; has-333@yamaguchi-u.ac.jp
}

Received 3 October 2014; Revised 18 December 2014; Accepted 21 December 2014

Academic Editor: Vikram Kate

Copyright (C) 2015 Hiroaki Shibata et al. This is an open access article distributed under the Creative Commons Attribution License, which permits unrestricted use, distribution, and reproduction in any medium, provided the original work is properly cited.

\begin{abstract}
Aim. To compare the efficacy of using computed tomography enteroclysis/enterography (CTE), capsule endoscopy (CE), and CTE with CE for diagnosing tumor lesions in the small intestine. Materials and Methods. We included 98 patients who underwent CE during the observation period and were subjected to CTE at our hospital from April 2008 to May 2014. Results. CTE had a significantly higher sensitivity than CE (84.6\% versus $46.2 \%, P=0.039)$, but there were no significant differences in specificity, positive or negative predictive values, or diagnostic accuracy rates. The sensitivity of CTE/CE was 100\%, again significantly higher than that of $\mathrm{CE}(P=0.002)$. The difference in specificity between $\mathrm{CTE} / \mathrm{CE}$ and $\mathrm{CE}$ was not significant, but there were significant differences in positive predictive values (100\% for CTE/CE versus $66.7 \%$ for CE, $P=0.012$ ), negative predictive values ( $100 \%$ versus $92.1 \%, P=0.008)$, and diagnostic accuracy rate $(100 \%$ versus $89.8 \%, P=0.001)$. The diagnostic accuracy rate was also significantly higher in CTE/CE versus CTE (100\% versus 95.9\%, $P=0.043)$. Conclusion. Our findings suggested that a combination of CTE and CE was useful for screening tumor lesions in the small intestine. This trial is registered with number UMIN000016154.
\end{abstract}

\section{Introduction}

In terms of frequency, small bowel tumors have been considered rare in comparison with gastric and colon tumors; they have accounted for approximately $6 \%$ of primary gastrointestinal tumors and only approximately $1 \%$ of malignant gastrointestinal tumors [1-4]. Since 2001, there has been a widespread use of new techniques for examining small intestines, such as capsule endoscopy (CE) and balloon endoscopy (BE), and, as a result, small intestine tumors were found to account for a higher percentage than previously reported [5-8].
CE was first reported by Iddan et al. and has become the first choice for screening lesions in the small intestine $[7,9]$. CE has mainly been reported to be useful in obscure gastrointestinal bleeding (OGIB) [10-12], but recent reports have also shown its usefulness in Crohn's disease [13-15]. Regarding neoplastic lesions, Ross et al. previously reported that the rate of diagnosing neoplastic tumor lesions in the small intestine by CE was 5/15 cases (33.3\%) [16]. Zagorowicz et al. previously reported that the passage of CE over neoplastic lesions present in the proximal jejunum was fast; therefore, there was a risk of overlooking the diagnosis [17]. Thus, even when $\mathrm{CE}$ is used, diagnosing tumor lesions in the small 
intestine can be difficult in some cases, and the prognosis of small intestine malignancies is unfavorable [18-20]. It would be preferable to have tests that accurately diagnose such tumor lesions.

Thus far, using computed tomography (CT) to perform evaluations on the small intestine has been considered impossible; however, CT enteroclysis/enterography (CTE) has made it possible, because the small intestine can be expanded by using a negative contrast agent and images are obtained through contrast-enhanced CT [21, 22]. CT enteroclysis is a testing method that consists of injecting a negative contrast agent into the small intestine through a cannula [21], whereas, in CT enterography, the small intestine is expanded through the oral ingestion of a negative contrast agent [22]. While most reports on CTE have been about its diagnostic performance for the intestinal inflammation and intestinal complications of Crohn's disease [23], reports on the utility of CTE in the diagnosis of tumor lesions in the small intestine have also been found recently [24]. However, to date, there have been few studies that compare CE to CTE for evaluating neoplastic lesions [25-27]; in addition, there has been no study on the tests' sensitivity or specificity of neoplastic lesions or their rate of diagnostic accuracy. Therefore, we compared the diagnostic performance of CTE with that of $\mathrm{CE}$ in the assessment of tumor lesions in the small intestine, and we also examined the diagnostic performance of using a combination of the two tests.

\section{Materials and Methods}

2.1. Patients. The study was conducted retrospectively on 98 CE-tested patients who had been subjected to CTE at our hospital between April 2008 and May 2014. The malefemale ratio was even (49/49 cases), and the mean age was $63.9 \pm 16.5$ years. The median duration of the time between $\mathrm{CTE}$ and $\mathrm{CE}$ was 2 days (range, $0-156$ days). The indications for the tests were as follows: OGIB for 73 patients (74.5\%); suspected neoplastic lesions for 12 (12.2\%); Crohn's disease for 2 (2.0\%); suspicion of Crohn's disease for 3 (3.1\%); and other for $8(8.2 \%)$ (Table 1). This study was approved by the Ethics Committee of Yamaguchi University Hospital.

2.2. Computed Tomography Enteroclysis/Enterography. CT enteroclysis was performed based on Liu et al.s method [28]. A nasal endoscope was inserted into the duodenum. Next, a guide wire was inserted into the jejunum from the forceps port of the endoscope. After the nasal endoscope was removed, a 16-French balloon-tipped tube was inserted into the duodenojejunal flexure along the guide wire. After the balloon at the tip of the tube was inflated, $1200-1800 \mathrm{~mL}$ of a polyethylene glycol (PEG) solution, which had been warmed to about $37^{\circ} \mathrm{C}$, was injected into the small intestine at $150 \mathrm{~mL} / \mathrm{min}$ by using a pump. After the PEG solution was injected, the patient was immediately moved to the CT device, and a plain CT was performed followed by a contrastenhanced CT. After the contrast agent for the contrastenhanced CT was injected, three-phase imaging ( $40 \mathrm{sec}$, $70 \mathrm{sec}$, and $120 \mathrm{sec}$ ) was performed. Before the patient was
TABLE 1: Characteristics of the patients participating in the study.

\begin{tabular}{lc}
\hline Number of cases & 98 \\
Sex, male (\%) & 50 \\
Age (years) & $63.9 \pm 16.5$ \\
$\mathrm{Hb}(\mathrm{g} / \mathrm{dL})$ at the time when CTE was performed & $9.4 \pm 3.3$ \\
Time interval (median value) between CTE and & $2 \pm 26.3$ \\
CE & \\
Purpose of the tests & 73 cases $(745 \%)$ \\
$\quad$ OGIB & 12 cases $(12.2 \%)$ \\
$\quad$ Suspicion of neoplastic lesions & 5 cases $(5.1 \%)$ \\
$\quad$ Inflammatory bowel disease & 8 cases $(8.2 \%)$ \\
$\quad$ Others &
\end{tabular}

$\mathrm{Hb}$ : hemoglobin; CTE: computed tomography enteroclysis/enterography; CE: capsule endoscopy; OGIB: obscure gastrointestinal bleeding.

moved to the CT device, butyl scopolamine $(20 \mathrm{mg}$ ) was injected intramuscularly as an antispasmodic agent. When butyl scopolamine was contraindicated, glucagon $(1 \mathrm{mg})$ was injected intramuscularly, and in patients for whom glucagon was also contraindicated, antispasmodic agents were not used. Tests using CT enterography were performed by referring to Huprich and Fletcher's method [29]. For 1h, 1000$1800 \mathrm{~mL}$ of the PEG solution was ingested orally, and CT imaging was performed by using the same method as that used during CT enteroclysis.

The CT images were interpreted by a radiologist (Kensaku Shimizu) with $>20$ years of experience in interpreting CT images. After a conventional interpretation was performed, the CTE findings were confirmed once again by examining the $\mathrm{CE}$ findings. The purpose of the test was to examine whether the lesions could be depicted by CTE, not to examine whether the physician could diagnose the small intestinal tumor by the CTE findings.

2.3. Capsule Endoscopy. All patients were given an explanation about the complications associated with $\mathrm{CE}$, such as an undescended or retained capsule, and were informed about the possible need for surgery in the case of a retained capsule, depending on the situation. Written informed consent was obtained for CE. The PillCam SB CE system (Given Imaging, Yokneam, Israel) was used, and the image interpretation was performed on a Rapid Reader (Version 6.5; Given Imaging). Each patient was instructed to fast for $12 \mathrm{~h}$ before the examination and was then asked to swallow the capsule. All patients were orally administered $40 \mathrm{mg}$ of dimethicone syrup with a small amount of water before the examination to reduce air bubbles. After the examination began, the patients were allowed to drink water after $2 \mathrm{~h}$ and to eat light meals until it was confirmed that the capsule had arrived at the small intestine as indicated on the real-time viewer. Video recorders were collected until it was confirmed that the capsule had arrived at the large intestine on the real-time viewer, and the data were transferred to a workstation for analysis. CE findings were determined by a digestive endoscopy specialist (Shinichi Hashimoto) who had experience in interpreting CE data for $>200$ cases. After a conventional interpretation was 
performed, the CE findings were confirmed once more by referencing the CTE findings. The purpose of the test was the same as previously described for CTE.

2.4. Method for Evaluating Tumor Lesions in the Small Intestine. A definitive diagnosis of the tumor lesions in the small intestine was determined on the basis of findings from histopathological tests conducted on resected or biopsy specimens collected from BE or surgery. For patients who did not undergo BE or surgery, the final diagnosis was determined on the basis of clinical and imaging findings. The criteria for negative tumor lesions in the small intestine were as follows: (1) no tumor was detected by CE or CTE; (2) the presence of the tumor lesions was negative in $\mathrm{BE}$ and surgical findings; and (3) the symptoms believed to be due to neoplastic lesions were absent for $\geq 3$ months after the tests.

2.5. Statistical Analysis. The chi-square test was used for the diagnostic rate of tumors. Statistical analysis was performed by using Ekuseru-Toukei 2012 (Social Survey Research Information Co., Ltd., Tokyo, Japan). Differences were considered statistically significant at a $P$ value of $<0.05$.

\section{Results}

3.1. CTE and CE Findings. With CTE, the rate of positive findings was 52/98 cases (53.1\%). Neoplastic lesions (i.e., polyps and submucosal tumors) were found in 13/98 cases (13.3\%); suspicion of angioectasia, arteriovenous malformation, and other vascular lesions was found in 23/98 (23.5\%); inflammatory findings (i.e., wall thickening, mucosal contrast enhancement, stenosis, fistulas, anal fistula, and perianal abscess) were found in 18/98 (18.4\%); other lesions (i.e., abnormal small intestinal transit, suspicion of diverticulum, and postoperative transformation) were found in 3/98 (3.1\%); and the findings were absent in 46/98 (46.9\%).

With CE, the rate of positive findings was 75/98 cases (76.5\%). Neoplastic lesions were found in $9 / 98$ cases $(9.2 \%)$; angioectasia was found in 25/98 (25.5\%), mucosal injuries (i.e., reddening, erosions, ulcers, and ulcer scars) were found in 48/98 (49.0\%); and the findings were absent in $23 / 98$ (23.5\%) (Table 2).

3.2. Small Bowel Tumor. Thirteen cases were finally diagnosed with neoplastic lesions, and in the remaining 85 , the presence of tumor lesions of the small intestine was not confirmed on the basis of their clinical course and the tests that were conducted subsequently. In 11 of 13 cases, the tumors of the small intestine were detectable by CTE, and, in 6, they were detectable by CE. In all cases, small bowel tumors were detected by CTE and CE. However, a false-positive result was found in 2 of 13 cases with diagnosis of neoplastic lesions detected by CTE and 3 of 9 cases detected by CE. Gastrointestinal stromal tumors (GISTs) were found in 5 cases and were the largest in number. Various types of tumors such as neuroendocrine tumors (NETs), ectopic pancreas, and capillary hemangiomas were detected, but the primary small intestine cancers were not detected (Table 3 ).
Figure 1 shows a case in which the tumor lesions (GISTs) were detected by both CTE and CE and were treated surgically. Figure 2 shows a case of Peutz-Jeghers syndrome; despite a tumor diameter $>5 \mathrm{~cm}$, the tumor could not be detected by CE. However, it was detected by CTE as a neoplastic lesion, and the tumor was then identified by BE; thus, polypectomy was performed.

The sensitivity to neoplastic lesions for CTE and CE was $84.6 \%$ and $46.2 \%$, respectively, which was significantly different $(P=0.039)$. The specificity for CTE and CE was $97.6 \%$ and $96.5 \%$, respectively $(P=0.650)$. The positive predictive values for CTE and CE were $84.6 \%$ and $66.7 \%$, respectively $(P=0.323)$. The negative predictive values were $97.6 \%$ and $92.1 \%$, respectively $(P=0.101)$, and the rates of diagnostic accuracy for CTE and CE were $95.9 \%$ and $89.8 \%$, respectively $(P=0.096)$. Although the CTE was better than $\mathrm{CE}$ for diagnosing neoplastic lesions, the only significant difference was in sensitivity (Table 4).

In addition, the results of the tests using a combination of CTE and CE (CTE/CE) were compared to those using $\mathrm{CE}$ alone, and the findings showed that CTE/CE had a $100 \%$ sensitivity while CE had a $46.2 \%$ sensitivity $(P=0.002)$, which was significantly different. Specificity for CTE/CE and CE was $100 \%$ and $96.5 \%$, respectively $(P=0.081)$. Although the specificity for CTE/CE was better than that for CE, the difference was not significant. The positive predictive values for $\mathrm{CTE} / \mathrm{CE}$ and $\mathrm{CE}$ were $100 \%$ and $66.7 \%$, respectively $(P=0.012)$; negative predictive values were $100 \%$ and $92.1 \%$, respectively $(P=0.008)$; and the diagnostic accuracy rates for CTE/CE and CE were $100 \%$ and $89.8 \%$, respectively $(P=0.001)$, all significant differences (Table 4). However, a comparison of the results from using a combination of CTE/CE to those from using CTE alone showed no significant difference in terms of sensitivity $(P=0.141)$, specificity $(P=0.151)$, positive predictive value $(P=0.141)$, or negative predictive value $(P=0.151)$. The diagnostic accuracy rates of CTE/CE and CTE were $100 \%$ and $95.9 \%$, respectively, which was significantly different $(P=0.043)$ (Table 4$)$.

\section{Discussion}

Compared to CE, our study findings showed that CTE had a higher performance for detecting tumor lesions in the small intestine. Additionally, the diagnosability of tumors increased even higher when CTE and CE were used together.

In our study, the sensitivity of CTE in the detection of small bowel tumor lesions was $11 / 13$ cases (84.6\%), and the sensitivity of CE was $6 / 13$ (46.2\%). These findings showed that CTE had a significantly higher sensitivity. A previous report by Hakim et al. also showed that the sensitivity of CTE in the detection of small bowel tumor lesions was 16/17 cases (94.1\%) and that of CE was 6/17 (35.3\%); therefore, CTE had a significantly higher sensitivity [26].

The particularly notable findings in this study were that, in as many as 7/13 cases (53.8\%), small bowel tumor lesions could not be detected by CE. Similarly, Johanssen et al. also reported that the sensitivity of $\mathrm{CE}$ in the detection of small bowel NETs was $3 / 8$ cases (37.5\%) [25]. Huprich et al. also reported that when they conducted CE on OGIB patients, 
TABLE 2: Computed tomography enteroclysis/enterography (CTE) and capsule endoscopy (CE) findings.

\begin{tabular}{|c|c|c|c|}
\hline \multicolumn{2}{|l|}{ CTE findings } & \multicolumn{2}{|l|}{ CE findings } \\
\hline Suspicion of angioectasia & 17 & Angioectasia & 25 \\
\hline Tumor mass in contact with the small intestine & 7 & Ulcers & 17 \\
\hline Enhanced contrast effect & 6 & Ulcer scars & 2 \\
\hline Wall thickening & 5 & Sores & 22 \\
\hline Stenosis & 3 & Reddening & 5 \\
\hline Vasodilatation & 3 & Suspicion of stenosis & 2 \\
\hline Suspicion of AVM & 2 & SMT & 6 \\
\hline Suspicion of vasculitis & 1 & Stagnation & 2 \\
\hline Suspicion of polyps & 1 & Polyps & 3 \\
\hline Tumorous lesions & 1 & Blood & 6 \\
\hline Densely stained punctiform lesion of the ileum & 1 & Diverticulum & 1 \\
\hline Pedunculated mass in the proximal jejunum & 1 & Lymphangiectasia & 1 \\
\hline Distension of the small intestine/abnormal small intestinal transit & 1 & No findings & 23 \\
\hline Nonstained tumor mass inside the small intestine & 1 & & \\
\hline Suspicion of diverticulum & 1 & & \\
\hline Pouchitis & 1 & & \\
\hline Postoperative changes in the small intestine & 1 & & \\
\hline Tumor mass in contact with the small intestine/transverse colon & 1 & & \\
\hline Fistula & 1 & & \\
\hline Perianal abscess & 1 & & \\
\hline Anal fistula & 1 & & \\
\hline No findings & 46 & & \\
\hline
\end{tabular}

AVM: arteriovenous malformations; SMT: submucosal tumor.

TABLE 3: Comparison of the computed tomography enteroclysis/enterography (CTE) and capsule endoscopy (CE) in terms of the final diagnoses.

\begin{tabular}{|c|c|c|c|c|c|}
\hline Number & Sex/age & CTE & $\mathrm{CE}$ & Final diagnosis & Reference standard \\
\hline 1 & $\mathrm{~F} / 55$ & No findings & Jejunal SMT & NET & $\mathrm{BE}$, liver metastasis \\
\hline 2 & $\mathrm{M} / 74$ & Tumorous lesion in the ileum & Sores, ulcers & Ectopic pancreas & $\mathrm{BE}$, surgery \\
\hline 3 & $\mathrm{~F} / 58$ & $\begin{array}{l}\text { Tumor mass in contact with the small } \\
\text { intestine }\end{array}$ & Angioectasia & Small intestine GIST & Surgery \\
\hline 4 & $\mathrm{~F} / 80$ & $\begin{array}{l}\text { Densely stained punctiform lesion in the } \\
\text { ileum }\end{array}$ & Sores, redness & Capillary hemangioma & $\mathrm{BE}$ \\
\hline 5 & $\mathrm{M} / 78$ & $\begin{array}{l}\text { Tumor mass in contact with the small } \\
\text { intestine }\end{array}$ & No findings & Small intestine GIST & $\mathrm{BE}$, surgery \\
\hline 6 & $\mathrm{~F} / 78$ & $\begin{array}{l}\text { Tumor mass in contact with the small } \\
\text { intestine }\end{array}$ & Sores, ulcers & Small intestine GIST & $\mathrm{BE}$, surgery \\
\hline 7 & $\mathrm{~F} / 25$ & Pedunculated mass in the jejunum & No findings & PJS & $\mathrm{BE}$ \\
\hline 8 & $\mathrm{~F} / 55$ & $\begin{array}{l}\text { Tumor mass in contact with the small } \\
\text { intestine }\end{array}$ & No findings & Small intestine GIST & $\mathrm{BE}$, surgery \\
\hline 9 & $\mathrm{M} / 72$ & $\begin{array}{l}\text { Nonstained tumor mass in the small } \\
\text { intestine }\end{array}$ & SMT & Lipoma & $\mathrm{BE}$, surgery \\
\hline 10 & $\mathrm{M} / 72$ & $\begin{array}{l}\text { Tumor mass in contact with the small } \\
\text { intestine }\end{array}$ & SMT & NET & $\mathrm{BE}$ \\
\hline 11 & $\mathrm{M} / 59$ & No findings & Polyps & Cronkhite-Canada syndrome & $\mathrm{CE}$ \\
\hline 12 & $\mathrm{~F} / 59$ & $\begin{array}{l}\text { Tumor mass in contact with the small } \\
\text { intestine }\end{array}$ & SMT & Small intestine GIST & $\mathrm{BE}$, surgery \\
\hline 13 & $\mathrm{M} / 87$ & $\begin{array}{l}\text { Tumor mass in contact with the small } \\
\text { intestine }\end{array}$ & SMT & Metastatic small bowel tumor & CTE, CE, anamnestic \\
\hline
\end{tabular}

SMT: submucosal tumor; NET: neuroendocrine tumors; GIST: gastrointestinal stromal tumor; PJS: Peutz-Jeghers' syndrome; BE: balloon endoscopy. 


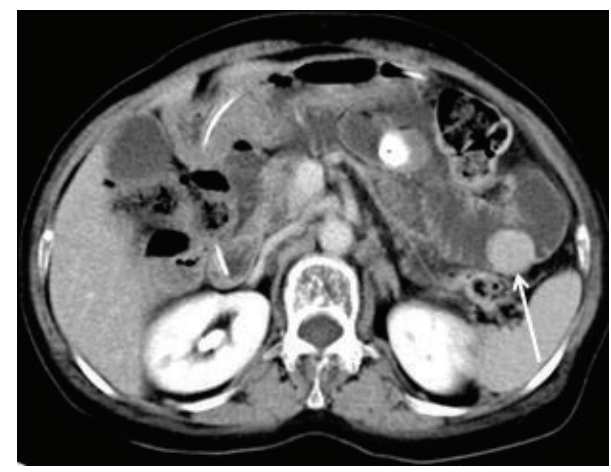

(a)

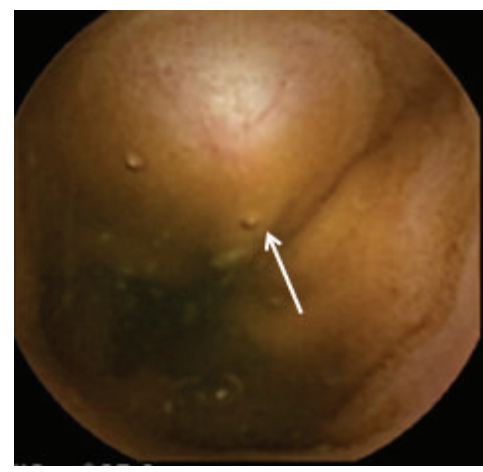

(b)

FIGURE 1: Findings from the computed tomography enteroclysis/enterography (CTE) and capsule endoscopy (CE). (a) Stained tumorous lesions in the small intestine detected by CTE and (b) submucosal tumor lesions confirmed by CE.

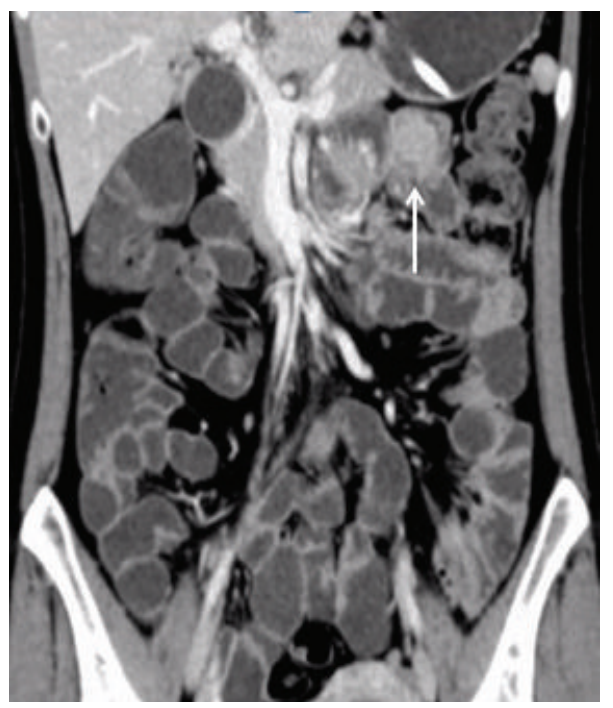

(a)

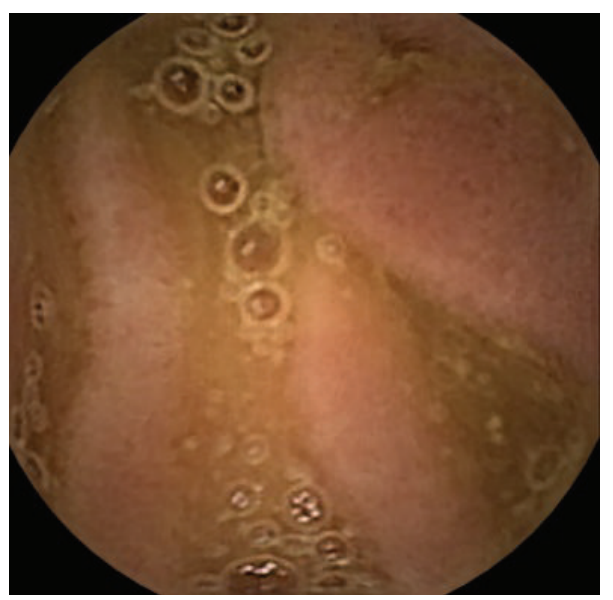

(c)

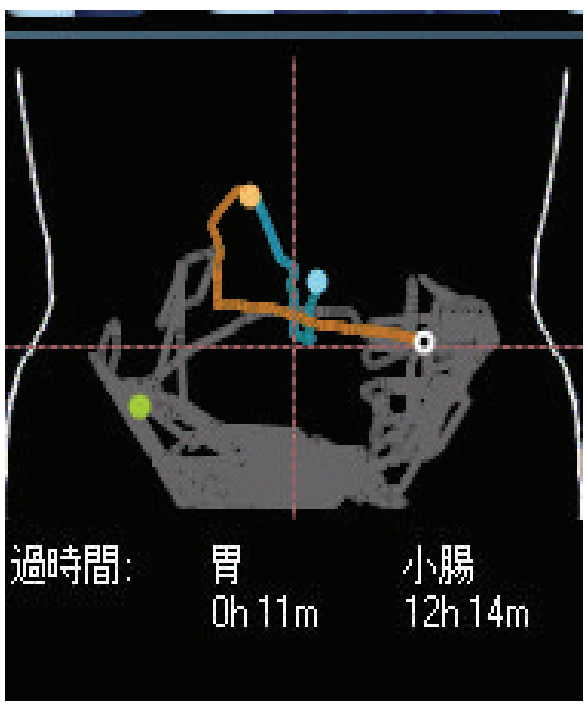

(b)



(d)

FIGURE 2: Findings from computed tomography enteroclysis/enterography (CTE), capsule endoscopy (CE), and balloon endoscopy (BE). (a) Strongly stained tumorous lesions in the small intestine detected by CTE; (b) the trajectory of CE in the vicinity of the area where CTE detected the lesions; (c) CE findings in the vicinity where lesions are detected by CTE. No lesion was detected by CE; (d) BE is performed after $\mathrm{CE}$, and polypoid lesion was confirmed in the vicinity of the lesion detected by CTE. 
TABLE 4: Comparison of computed tomography enteroclysis/enterography (CTE) and capsule endoscopy (CE) in terms of detecting the neoplastic lesions.

\begin{tabular}{|c|c|c|c|c|c|}
\hline & CTE & & $\mathrm{CE}$ & & CTE/CE \\
\hline & & $P=0.039^{*}$ & & $P=0.002^{*}$ & \\
\hline \multirow[t]{3}{*}{ Sensitivity } & $84.6 \%$ & & $46.2 \%$ & & $100 \%$ \\
\hline & & & $P=0.141$ & & \\
\hline & & $P=0.650$ & & $P=0.081$ & \\
\hline \multirow[t]{3}{*}{ Specificity } & $97.6 \%$ & & $96.5 \%$ & & $100 \%$ \\
\hline & & & $P=0.155$ & & \\
\hline & & $P=0.323$ & & $P=0.012^{*}$ & \\
\hline \multirow[t]{3}{*}{ Positive predictive value } & $84.6 \%$ & & $66.7 \%$ & & $100 \%$ \\
\hline & & & $P=0.141$ & & \\
\hline & & $P=0.101$ & & $P=0.008^{*}$ & \\
\hline \multirow[t]{3}{*}{ Negative predictive value } & $97.6 \%$ & & $92.1 \%$ & & $100 \%$ \\
\hline & & & $P=0.155$ & & \\
\hline & & $P=0.096$ & & $P=0.001^{*}$ & \\
\hline \multirow[t]{2}{*}{ Rate of diagnostic accuracy } & $95.9 \%$ & & $89.8 \%$ & & $100 \%$ \\
\hline & & & $P=0.043^{*}$ & & \\
\hline
\end{tabular}

${ }^{*}$ Significant differences.

including 9 with small bowel tumor lesions, the diagnosis of small bowel tumors was overlooked in $3 / 9$ cases $(33.3 \%)$; therefore, they reported that, in some cases, small bowel tumor lesions can be overlooked by CE [30]. Thus, although $\mathrm{CE}$ has been considered as the first choice procedure for screening small bowel diseases [7,9], its low sensitivity in the detection of small bowel tumor lesions may cause them to be overlooked. As a result of this, the best timing for therapeutic intervention may be missed.

Our study showed that, with CTE, we were able to detect $7(100 \%)$ of the 7 cases in which small bowel lesions could not be detected with $\mathrm{CE}$; meanwhile, with $\mathrm{CE}$, we were able to detect $2(100 \%)$ of the 2 cases in which neoplastic lesions could not be detected with CTE, suggesting that the combined use of CTE and CE would allow their abilities to complement each other and that the rate of overlooked small bowel tumor lesions using $\mathrm{CE}$ can be greatly reduced by combining CE with CTE.

No difference was found between CTE and CE in terms of specificity, positive predictive value, negative predictive value, or rate of diagnostic accuracy; however, the sensitivity with CTE was significantly higher than with CE.

A comparison of tests using a combination of CTE and CE to tests using $\mathrm{CE}$ alone revealed that a combination of CTE and CE showed greater significant differences in sensitivity, positive predictive value, negative predictive value, and rate of diagnostic accuracy. Therefore, in comparison with using CE alone for screenings, a combination of CE and CTE may be more highly reliable in diagnosing small bowel tumor lesions.

Our study also showed that small bowel tumor lesions might be missed by CE. It especially has been reported that for lesions in the jejunum in which a capsule moves quickly, submucosal tumors are easily missed [17, 31-34]. The issue that tumor detection depends on the transit rate may be resolved by the use of PillCam SB3, which automatically regulates the number of images according to the progress rate of capsules in the small bowel [35]. However, it is thought that submucosal tumors that grow outside the intestine may be difficult to detect by CE. By solving this issue, CTE may complement CE. However, there is the problem of radiation exposure in CTE. As less invasive tests to evaluate small bowel tumors, magnetic resonance enterography (MRE) and ultrasonography (USG) have been reported as useful to detect small bowel tumor-related lesions [36-38]. Nevertheless, various problems exist that MRE takes more time in comparison with CTE and diagnosis by USG depends on the skill of the diagnostician. Therefore, the prevalence of their use is lower than that of CTE and CE. Further examination using these modalities will be required in the future.

In addition, although BE can detect small bowel tumors, its performance for diagnosing is thought to be equivalent to CE. Furthermore, as a screening test, $\mathrm{BE}$ is a highly invasive procedure $[34,39]$.

The limitations of this study were the fact that it was retrospective and the number of cases was small. In addition, with regard to the rate of detection of neoplastic lesions by $\mathrm{CE}$, an existing report used $\mathrm{CE}$ on 443 subjects, which detected small bowel tumors in 11 of the subjects (2.4\%) [40]. In our facility, the number of small bowel tumors detected was as high as $6 / 98(6.1 \%)$ and small intestine cancers, which accounted for $33-47 \%$ of primary small intestine tumors $[4,41]$, and malignant lymphomas, which accounted for 10$30 \%$ of malignant tumors of the small intestine [42], were not included in the details regarding the small bowel tumor lesions that were detected. Thus, the cases that were selected in our study may have been biased. Lastly, not all the cases underwent total enteroscopy using BE or surgical treatment; therefore, the findings pertaining to specificity, positive and negative predictive values, and rate of diagnostic accuracy maybe different from the actual situation.

However, to our knowledge, no report has mentioned the specificity, positive predictive value, negative predictive 
value, or rate of diagnostic accuracy with regard to the detecting small bowel tumor lesions. Therefore, our study is the first to examine this topic. Our findings indicate a need to conduct multicenter prospective studies in the future to further increase the diagnostic accuracy of small bowel tumor lesions.

\section{Conclusions}

Our study findings showed that the sensitivity for CTE in the detection of small bowel tumor lesions was significantly higher than that of $\mathrm{CE}$. In comparison with the tests performed using CE alone, the combination of CTE and CE resulted in significant differences in terms of sensitivity, positive predictive value, negative predictive value, and rate of diagnostic accuracy. This suggests that CTE should be the first line of investigation in the screening for small bowel tumor and may be followed, if required, by $\mathrm{CE}$.

\section{Conflict of Interests}

The authors declare that there is no conflict of interests regarding the publication of this paper.

\section{References}

[1] R. de Franchis, E. Rondonotti, C. Abbiati, G. Beccari, and C. Signorelli, "Small bowel malignancy," Gastrointestinal Endoscopy Clinics of North America, vol. 14, no. 1, pp. 139-148, 2004.

[2] S. S. Gill, D. M. Heuman, and A. A. Mihas, "Small intestinal neoplasms," Journal of Clinical Gastroenterology, vol. 33, no. 4, pp. 267-282, 2001.

[3] J. A. DiSario, R. W. Burt, H. Vargas, and W. P. McWhorter, "Small bowel cancer: epidemiological and clinical characteristics from a population-based registry," The American Journal of Gastroenterology, vol. 89, no. 5, pp. 699-701, 1994.

[4] T. Yao, K. Yao, H. Matake et al., "Tumors of the small intestine: summary of Yaocases reported in Japan during the recent fiveyear period (1995-1999)," Stomach and Intestine, vol. 36, pp. 871881, 2001.

[5] H. Yamamoto, Y. Sekine, Y. Sato et al., "Total enteroscopy with a nonsurgical steerable double-balloon method," Gastrointestinal Endoscopy, vol. 53, no. 2, pp. 216-220, 2001.

[6] H. Yamamoto, H. Kita, K. Sunada et al., "Clinical outcomes of double-balloon endoscopy for the diagnosis and treatment of small-intestinal diseases," Clinical Gastroenterology and Нераtology, vol. 2, no. 11, pp. 1010-1016, 2004.

[7] G. Iddan, G. Meron, A. Glukhovsky, and P. Swain, "Wireless capsule endoscopy," Nature, vol. 405, no. 6785, pp. 417-418, 2000.

[8] K. Mitsui, S. Tanaka, H. Yamamoto et al., "Role of doubleballoon endoscopy in the diagnosis of small-bowel tumors: the first Japanese multicenter study," Gastrointestinal Endoscopy, vol. 70, no. 3, pp. 498-504, 2009.

[9] R. Eliakim, "Video capsule endoscopy of the small bowel," Current Opinion in Gastroenterology, vol. 29, no. 2, pp. 133-139, 2013.

[10] Z. Fireman and Y. Kopelman, "The role of video capsule endoscopy in the evaluation of iron deficiency anaemia," Digestive and Liver Disease, vol. 36, no. 2, pp. 97-102, 2004.
[11] S. Mazzarolo and P. Brady, "Small bowel capsule endoscopy: a systematic review," Southern Medical Journal, vol. 100, no. 3, pp. 274-280, 2007.

[12] B. M. Graça, P. A. Freire, J. B. Brito, J. M. Ilharco, V. M. Carvalheiro, and F. Caseiro-Alves, "Gastroenterologic and radiologic approach to obscure gastrointestinal bleeding: how, why, and when?" Radiographics, vol. 30, no. 1, pp. 235-252, 2010.

[13] B. S. Lewis, G. M. Eisen, and S. Friedman, "A pooled analysis to evaluate results of capsule endoscopy trials," Endoscopy, vol. 37, no. 10, pp. 960-965, 2005.

[14] P. Swain, "Wireless capsule endoscopy and Crohn's disease," Gut, vol. 54, no. 3, pp. 323-326, 2005.

[15] S. L. Triester, J. A. Leighton, G. I. Leontiadis et al., "A metaanalysis of the yield of capsule endoscopy compared to other diagnostic modalities in patients with non-stricturing small bowel Crohn's disease," The American Journal of Gastroenterology, vol. 101, no. 5, pp. 954-964, 2006.

[16] A. Ross, S. Mehdizadeh, J. Tokar et al., "Double balloon enteroscopy detects small bowel mass lesions missed by capsule endoscopy," Digestive Diseases and Sciences, vol. 53, no. 8, pp. 2140-2143, 2008.

[17] E. S. Zagorowicz, A. M. Pietrzak, E. Wronska et al., "Small bowel tumors detected and missed during capsule endoscopy: single center experience," World Journal of Gastroenterology, vol. 19, no. 47, pp. 9043-9048, 2013.

[18] J. S. Chow, C. C. Chen, H. Ahsan, and A. I. Neugut, "A population-based study of the incidence of malignant small bowel tumours: SEER, 1973-1990," International Journal of Epidemiology, vol. 25, no. 4, pp. 722-728, 1996.

[19] N. H. Wright, M. Pennazio, J. R. Howe et al., "Carcinoma of the small intestine," in World Health Organization Classification of Tumors. Pathology and Genetics of Tumors of the Digestive System, S. R. Hamilton and L. A. Aaltonen, Eds., pp. 70-74, IARC Press, Lyon, France, 2000.

[20] M. S. Talamonti, L. H. Goetz, S. Rao, and R. J. Joehl, "Primary cancers of the small bowel: analysis of prognostic factors and results of surgical management," Archives of Surgery, vol. 137, no. 5, pp. 564-570, 2002.

[21] R. Klöppel, J. Thiele, and J. Bosse, "The sellink CT method," RoFo, vol. 156, no. 3, pp. 291-292, 1992.

[22] P. Reittner, T. Goritschnig, W. Petritsch et al., "Multiplanar spiral CT enterography in patients with Crohn's disease using a negative oral contrast material: initial results of a noninvasive imaging approach," European Radiology, vol. 12, no. 9, pp. 22532257, 2002.

[23] P. B. Wold, J. G. Fletcher, C. D. Johnson, and W. J. Sandborn, "Assessment of small bowel Crohn disease: noninvasive peroral CT enterography compared with other imaging methods and endoscopy-feasibility study," Radiology, vol. 229, no. 1, pp. 275281, 2003.

[24] P. Soyer, M. Aout, C. Hoeffel, E. Vicaut, V. Placé, and M. Boudiaf, "Helical CT-enteroclysis in the detection of smallbowel tumours: a meta-analysis," European Radiology, vol. 23, no. 2, pp. 388-399, 2013.

[25] S. Johanssen, M. Boivin, H. Lochs, and W. Voderholzer, "The yield of wireless capsule endoscopy in the detection of neuroendocrine tumors in comparison with CT enteroclysis," Gastrointestinal Endoscopy, vol. 63, no. 4, pp. 660-665, 2006.

[26] F. A. Hakim, J. A. Alexander, J. E. Huprich, M. Grover, and F. T. Enders, "CT-enterography may identify small bowel tumors not detected by capsule endoscopy: eight years experience at Mayo 
Clinic Rochester," Digestive Diseases and Sciences, vol. 56, no. 10, pp. 2914-2919, 2011.

[27] S. Khalife, P. Soyer, A. Alatawi et al., "Obscure gastrointestinal bleeding: preliminary comparison of 64-section CT enteroclysis with video capsule endoscopy," European Radiology, vol. 21, no. 1, pp. 79-86, 2011.

[28] Y. B. Liu, C. H. Liang, Z. L. Zhang et al., "Crohn disease of small bowel: multidetector row CT with CT enteroclysis, dynamic contrast enhancement, CT angiography, and 3D imaging," Abdominal Imaging, vol. 31, no. 6, pp. 668-674, 2006.

[29] J. E. Huprich and J. G. Fletcher, "CT enterography: principles, technique and utility in Crohn's disease," European Journal of Radiology, vol. 69, no. 3, pp. 393-397, 2009.

[30] J. E. Huprich, J. G. Fletcher, J. L. Fidler et al., "Prospective blinded comparison of wireless capsule endoscopy and multiphase CT enterography in obscure gastrointestinal bleeding," Radiology, vol. 260, no. 3, pp. 744-751, 2011.

[31] A. K. H. Chong, B. W. K. Chin, and C. G. Meredith, "Clinically significant small-bowel pathology identified by double-balloon enteroscopy but missed by capsule endoscopy," Gastrointestinal Endoscopy, vol. 64, no. 3, pp. 445-449, 2006.

[32] M. M. Baichi, R. M. Arifuddin, and P. S. Mantry, "Small-bowel masses found and missed on capsule endoscopy for obscure bleeding," Scandinavian Journal of Gastroenterology, vol. 42, no. 9, pp. 1127-1132, 2007.

[33] S. Fujimori, T. Seo, K. Gudis et al., "Diagnosis and treatment of obscure gastrointestinal bleeding using combined capsule endoscopy and double balloon endoscopy: 1-year follow-up study," Endoscopy, vol. 39, no. 12, pp. 1053-1058, 2007.

[34] A. Fukumoto, S. Tanaka, T. Shishido, Y. Takemura, S. Oka, and K. Chayama, "Comparison of detectability of smallbowel lesions between capsule endoscopy and double-balloon endoscopy for patients with suspected small-bowel disease," Gastrointestinal Endoscopy, vol. 69, no. 4, pp. 857-865, 2009.

[35] E. Redondo-Cerezo, A. D. Sánchez-Capilla, P. de La TorreRubio, and J. de Teresa, "Wireless capsule endoscopy: perspectives beyond gastrointestinal bleeding," World Journal of Gastroenterology, vol. 20, no. 42, pp. 15664-15673, 2014.

[36] G. Masselli and G. Gualdi, "CT and MR enterography in evaluating small bowel diseases: when to use which modality?" Abdominal Imaging, vol. 38, no. 2, pp. 249-259, 2013.

[37] E. Amzallag-Bellenger, P. Soyer, C. Barbe, M.-D. Diebold, G. Cadiot, and C. Hoeffel, "Prospective evaluation of magnetic resonance enterography for the detection of mesenteric small bowel tumours," European Radiology, vol. 23, no. 7, pp. 19011910, 2013.

[38] R. Kralik, P. Trnovsky, and M. Kopáčová, “Transabdominal ultrasonography of the small bowel," Gastroenterology Research and Practice, vol. 2013, Article ID 896704, 11 pages, 2013.

[39] N. Ohmiya, M. Nakamura, I. Hirata et al., "Management of small-bowel polyps at double-balloon enteroscopy," Annals of Translational Medicine, vol. 2, no. 3, article 30, 2014.

[40] D. Urbain, D. de Looze, I. Demedts et al., "Video capsule endoscopy in small-bowel malignancy: a multicenter Belgian study," Endoscopy, vol. 38, no. 4, pp. 408-411, 2006.

[41] F. Miao, M. L. Wang, and Y. H. Tang, "New progress in CT and MRI examination and diagnosis of small intestinal tumors," World Journal of Gastrointestinal Oncology, vol. 2, no. 5, pp. 222228, 2010.

[42] S. Nakamura, K. Matsumoto, T. Yao et al., "Tumors of the small intestine: malignant lymphomas," Stomach and Intestine, vol. 43, pp. 533-538, 2008. 




The Scientific World Journal
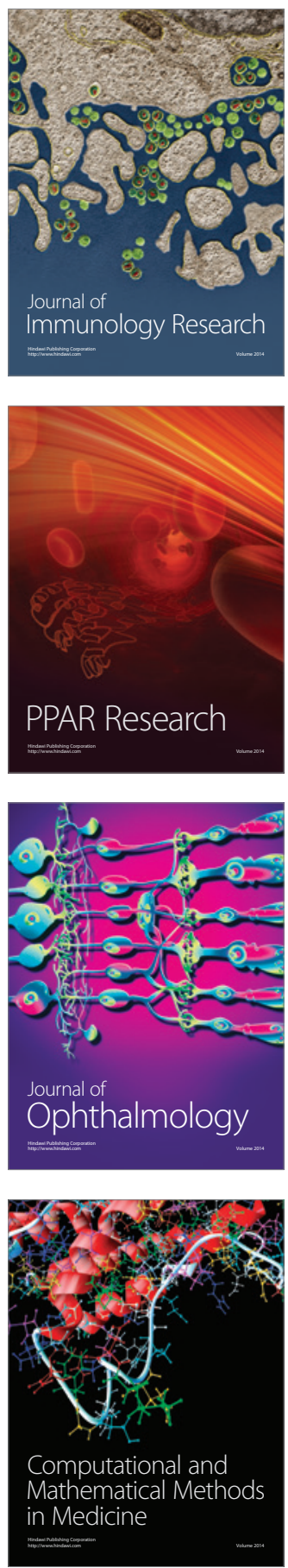

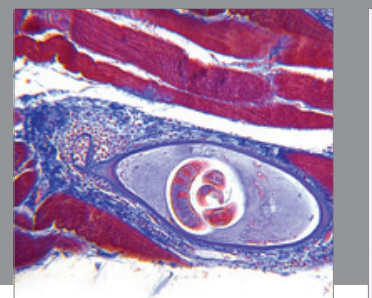

Gastroenterology

Research and Practice
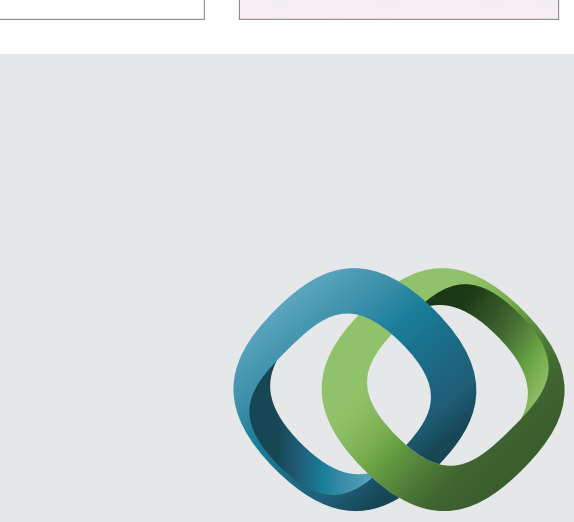

\section{Hindawi}

Submit your manuscripts at

http://www.hindawi.com
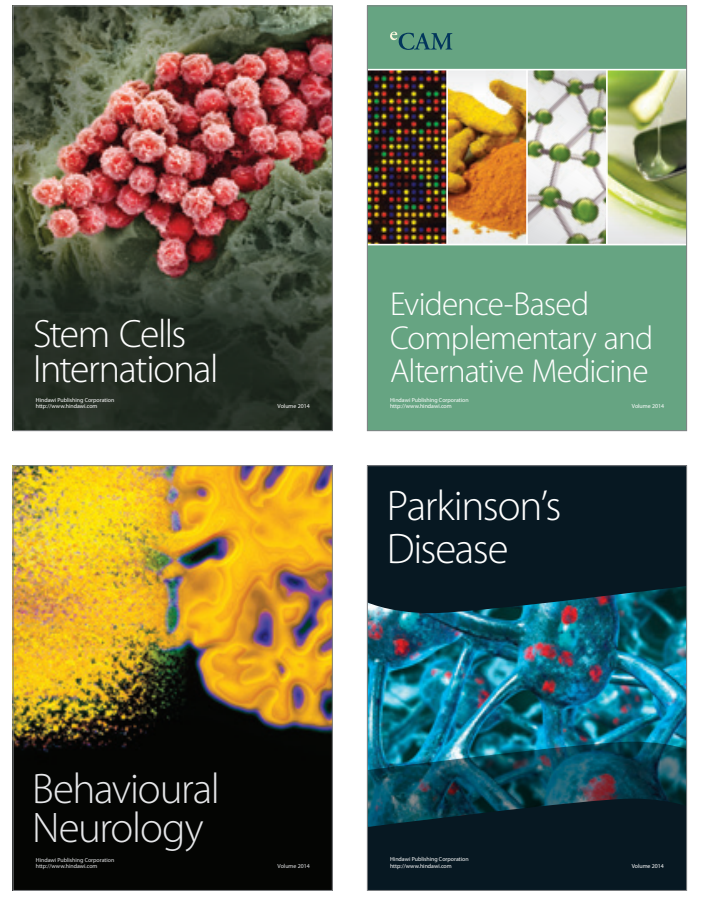
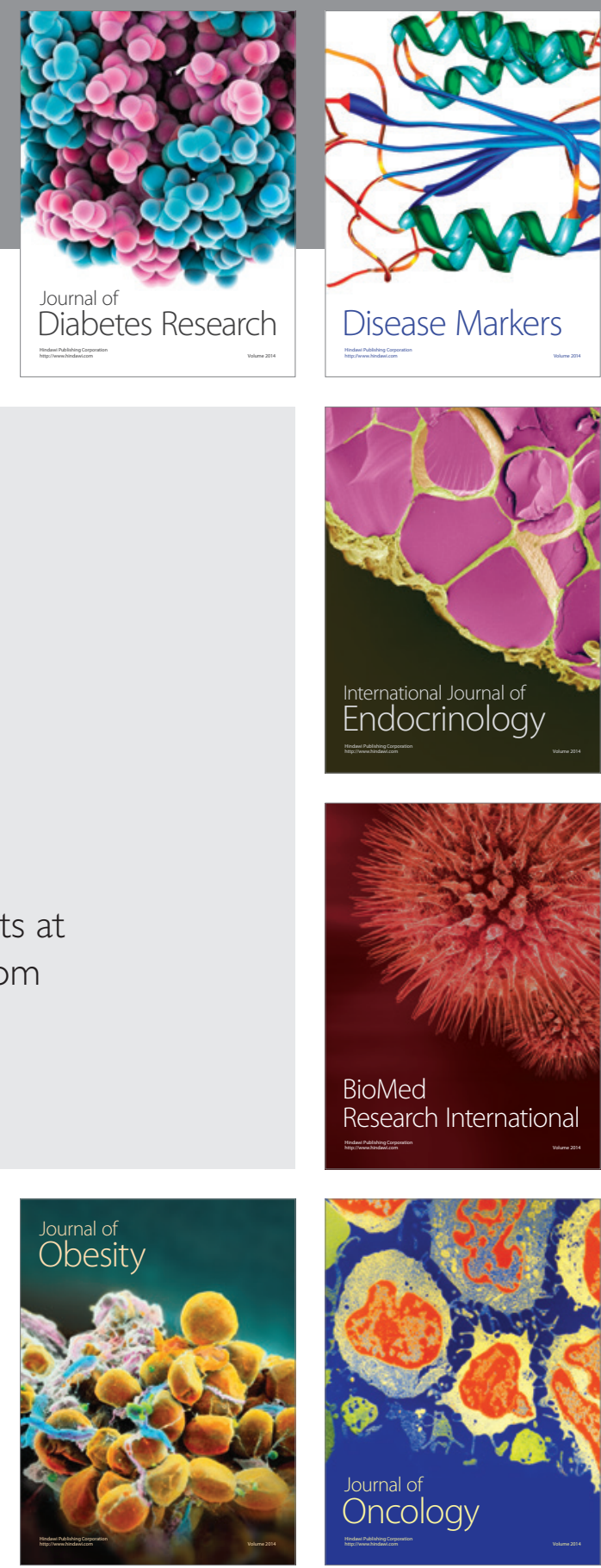

Disease Markers
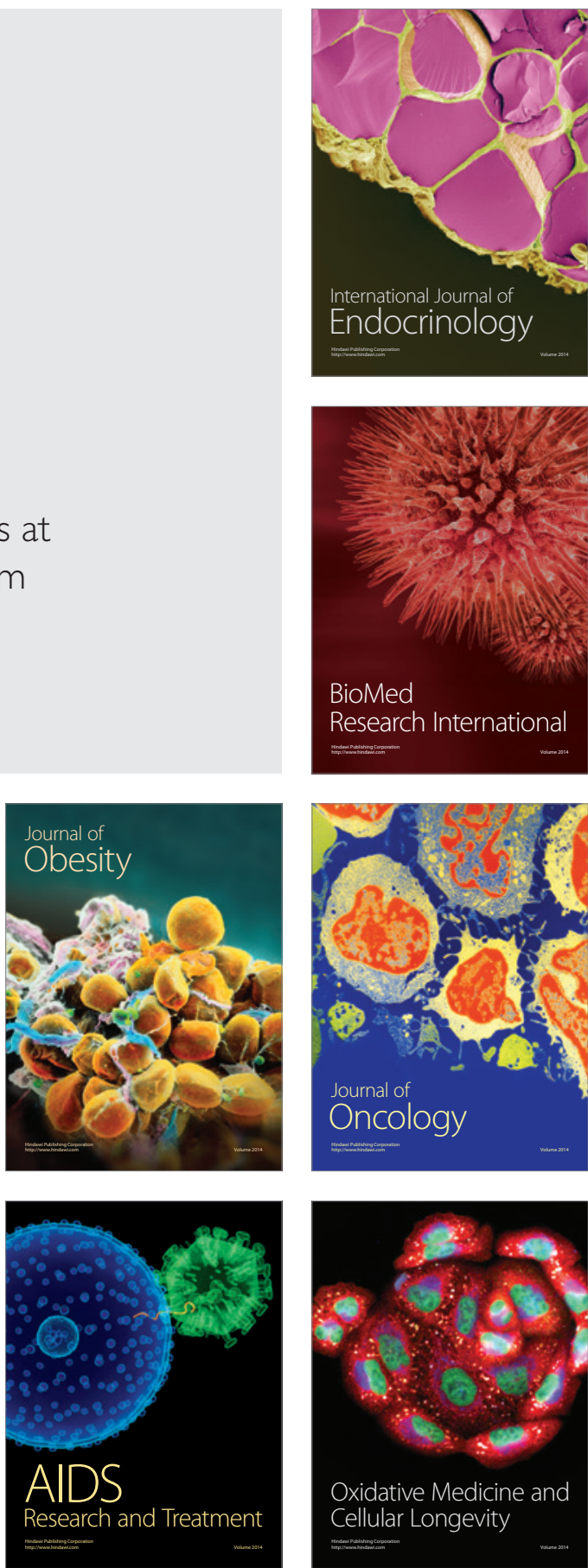